\title{
Cytokine detection for the diagnosis of chromium allergy ${ }^{*}$ \\ Detecção de citocinas no diagnóstico de pacientes alérgicos ao cromo
}

\author{
Luis Eduardo Agner Machado Martins ${ }^{1}$
}

Vitor Manoel Silva dos Reis ${ }^{2}$

DOI: $h$ ttp://dx.doi.org/10.1590/abd1806-4841.20132164

\begin{abstract}
BACKGROUND: Patch testing remains the gold standard method for the identification of the etiologic agent of allergic contact dermatitis. However, it is a subjective, time-consuming exam whose technique demands special care and which presents some contraindications, which hamper its use. In a recent study, we showed that the proliferation assay can suitably replace patch testing for the diagnosis of chromium allergy, which had been previously demonstrated only for nickel allergy. In this study, we try to refine the method by reducing the incubation period of cultures for lymphocyte proliferation assays in response to chromium. ОвјестіvE: Develop an alternative or complementary diagnostic test for chromium allergic contact dermatitis. METHODS: We compared the production of 9 cytokines (IFN-Y, IL-2, IL-4, IL-5, IL-10, IL-12, IL-13, IL-17 and RANTES) between 18 chromium-allergic patients and 19 controls. ResulTs: Chromium increased the production of IFN-y, IL-5, IL-2 and IL-13 in allergic patients, but only IL-2 and especially IL-13 helped discriminate allergic patients from controls. The sensitivity, specificity and accuracy found with IL-13 were about $80 \%$. ConCLUSIONS: IL-13 and IL-2 detection may be used to diagnose chromium allergy in 2-day cultures. However, in general, the 6-day cultures seem to be superior for this purpose.
\end{abstract}

Keywords: Chromium; Cytokines; Dermatitis, allergic contact; Dermatitis, contact; Laboratory test; Lymphocyte activation

Resumo: FUnDAMENTOS: O teste de contato permanece como padrão ouro para a identificação do agente causal da dermatite de contato alérgica, mas é um exame subjetivo, que demanda considerável tempo do paciente e do medico, exige cuidados na sua técnica e apresenta algumas contra-indicações que dificultam o seu uso. Em um estudo recente demonstramos que o teste de proliferação pode adequadamente substituir o teste de contato no diagnóstico de alergia ao cromo, algo previamente demonstrado apenas para o níquel. Neste estudo tentamos refinar o método reduzindo o período de incubação das culturas do teste de proliferação para o cromo. OBjETIVo: Desenvolver um método alternativo ao teste de contato para o diagnóstico dermatite de contato alérgica ao cromo. MÉTodos: Comparamos o estímulo provocado pelo cromo na produção de nove citocinas (IFN- $\gamma$, IL2, IL-4, IL-5, IL-10, IL-12, IL-13, IL-17 e RANTES) no sobrenadante das culturas de células do sangue periférico de 18 pacientes alérgicos ao cromo e 19 controles. RESULTADOS: O cromo aumentou a produção de IFN-y, IL-5, IL-2 e IL-13, mas apenas as citocinas IL-2 e principalmente IL-13 foram capazes de discriminar pacientes de controles. A sensibilidade, especificidade e acurácia encontradas com a IL-13 foram de aproximadamente de 80\%. ConCLusõEs: Concluímos que a detecção de IL-2 e IL-13 podem ser útil para o diagnóstico de alergia a cromo na cultura de 2 dias. Todavia, as culturas de 6 dias parecem, de um modo geral, superiores as de 2 dias para esse fim.

Palavras-chave: Ativação linfocitária; Citocinas; Cromo; Dermatite alérgica de contato; Dermatite de contato; Testes laboratoriais

Received on 30.09.2012.

Approved by the Advisory Board and accepted for publication on 18.11.2012.

* Work conducted at the Outpatient Clinic of Dermatology, Clinics Hospital, and at the Laboratory of Medical Investigation in Dermatology and Immunodeficiencies, Faculty of Medicine of the University of São Paulo (FMUS) - São Paulo, Brazil.

Financial Support: None

Conflict of Interest: None.

Professor of Dermatology at Hospital Universitário Evangélico de Curitiba - Faculdade Evangélica do Paraná (HUEC-FEPAR) - Curitiba (PR), Brazil.

PhD in Dermatology - Professor of Dermatology, Departament of Dermatology, Clinics Hospital, Faculty of Medicine of the University of São Paulo (Hospital de Clínicas da Faculdade de Medicina da Universidade São Paulo - HC-FMUSP) - São Paulo (SP), Brazil.

(C)2013 by Anais Brasileiros de Dermatologia 


\section{INTRODUCTION}

Chromium-induced allergic contact dermatitis (ACD) is very important, especially in the context of occupational dermatitis. It mainly affects construction workers who deal with cement on a daily basis. ${ }^{1-4}$

The tests currently used for the diagnosis of ACD are the patch tests (PT), which have already been established in routine medical practice. These tests are very useful, but they have their known disadvantages. ${ }^{5.9}$ Among these, it is clear that PT for metal allergy can lead to false-positive reactions of an irritant nature. ${ }^{10}$ In the United States, the concentration of potassium dichromate in the PT has been reduced from $0.5 \%$ to $0.25 \%$ to decrease irritant reactions. However, a reduced concentration leads to decreased sensitivity. For this reason, this agent continues to be tested at a concentration of $0.5 \%$ in Europe, but special attention is given to the relevance of the results..$^{11,12}$

The importance of the etiologic diagnosis in the prognosis of ACD together with the limitations and difficulties inherent to the PT point to the need for developing a diagnostic test that is less dependent on the presence of the patient and physician. Despite all these drawbacks, patch tests are still largely used, and their advantages are far from being overcome. However, it is worth trying an alternative, and the most promising one is the lymphocyte proliferation assay (LPA), since this exam is based on peripheral blood samples, collected as in any other laboratory test. This method consists in exposing the patient's lymphocytes to the suspected allergen., ${ }^{3,13}$ Based on the new concepts of the pathogenesis of $A C D$, we should detect differences between the lymphocytes of sensitized and non-sensitized patients, since the inflammation in ACD results from the action of previously sensitized lymphocytes and of memory, effector or regulatory lymphocytes, all of them with a selective production of cytokines. ${ }^{3,13}$ Among these differences, there is increased number of these cells (blastic transformation), cytokine proliferation and production; all of these changes can be measured., ${ }^{3,13,14}$ No matter what the method is, the cells are cultivated in the presence and in the absence of the antigen, and the results found in the stimulated and unstimulated cultures are compared. ${ }^{3,13,14}$

Blastic transformation is measured by the proportion of blastic cells in the cultures, proliferation is measured by the rate of incorporation of $3 \mathrm{H}$ thymidine, and the production of cytokines is measure by their quantification. The determination of the percentage of blastic cells is a laborious and subjective process. It was first replaced by lymphocyte proliferation rate and then by the detection of cytokines. The ratio and difference found between stimulated and unstimulated cultures, known as stimulation index (SI) and delta $(\varnothing)$, respectively, are the most commonly used parameters as a stimulus-response criterion., ${ }^{3,13,14}$

Response detection by LPA had shown, until recently, adequate and reproducible results only for the diagnoses of allergy to nickel. ${ }^{13}$ Our group has carried out research with lymphocyte cultures, assessing its proliferation in response to nickel and chromium, confirming the appropriate results for nickel and the already described low sensitivity to chromium..$^{13,14}$ Recently analyzing cytokine production in cultures from chromium-allergic patients, we have been able to demonstrate that the amount of IL-13 is substantially higher in these cultures, offering great accuracy for this diagnosis. ${ }^{14}$ Our group carried out all of these experiments in six-day cultures. As the production and release of cytokines happen quickly, the duration of the culture can be reduced, accelerating the process. In addition, different culture times may show different cytokine profiles, bringing new sensitivity, specificity and accuracy values and expanding the diagnostic capacity of the method. To evaluate these possibilities, this study was performed with 48 -hour cultures.

\section{MATERIAL AND METHODS Patients and controls}

Thirty-seven patients were selected at the Outpatient Clinic of Dermatology of the Clinics Hospital, Faculty of Medicine, University of São Paulo, between November 2009 and December 2010. Eighteen patients (17 men and 01 woman aged 36-65 years old, a mean age of 50 years) with chromium ACD proven by anamnesis, physical examination and recent patch testing (within 2 years) constituted the group of cases. The control group was formed by 19 patients (11 men and 8 women aged between 26 and 68 years, a mean age of 44 years) with ACD caused by other allergens, with no history or physical examination compatible with the diagnosis of allergy to chromium and with negative patch test results to chromium. An informed consent, approved by the Ethics Committee, was obtained from all participants.

\section{Patch test}

All patients were tested with the Brazilian standard battery (IPI-ASAC Brasil ${ }^{\oplus}$, Rio de Janeiro, Brazil), composed of 30 antigens, including potassium dichromate $0.5 \%$ in petrolatum. The allergens were applied to the upper back with the help of chambers and appropriate adhesive tape (3M, St. Paul, MN, USA) for 2 days. The results were read in 48 and 96 hours, and the reactions were registered in accordance with the criteria established by the International Contact Dermatitis Research Group: + = erythema and infiltration, $++=$ erythema, infiltration and vesicles, and ++ 
$+=$ confluent vesicles, erythema and infiltration.

\section{Lymphocyte proliferation assay in chromium con- tact sensitivity}

It was possible to isolate mononuclear cells, monocytes and lymphocytes from other blood elements through the centrifugation of peripheral venous blood with the help of a density gradient (Ficoll-Hypaque). These cells were washed and suspended $\left(2 \times 10^{6}\right.$ cells $\left./ \mathrm{mL}\right)$ in RPMI 1640 culture medium supplemented with gentamicin and $10 \%$ of human $\mathrm{AB}$ serum. Aliquots $(100 \mu \mathrm{L})$ of this suspension were cultured with $100 \mu \mathrm{L}$ of RPMI 1640 culture medium (unstimulated cultures) or with $100 \mu \mathrm{L}$ of a solution of chromium chloride $666 \mu \mathrm{L} / \mathrm{ml}$ $\left(\mathrm{CrCl}_{3} 6 \mathrm{H}_{2} \mathrm{O}\right.$; purity $\geq 96 \%$, Merck, Darmstadt, Germany) prepared in RPMI 1640 culture medium (stimulated cultures), as previously described. ${ }^{3,14}$ The cultures for the detection of cytokines were performed in monoplicate in 96-well flat bottom plates (TPP ${ }^{\circledR}$, Switzerland, Europe) with an incubation period of two days. Another culture in triplicate was simultaneously prepared as a positive control, with $5 \mu \mathrm{g} / \mathrm{mL}$ of pokeweed (Phytolacca americana; Sigma, St Louis, MO, USA), a non-specific lymphocyte activator. The incubation period of the control cultures was 6 days, and the response was measured by detecting $3 \mathrm{H}$ thymidine.

\section{Detection of cytokines}

The expression of the nine cytokines (IFN-, IL2, IL-4, IL-5, IL-10, IL-12, IL-13, IL-17 and RANTES) was measured in the supernatant of cultures from patients and controls. After an incubation period of 2 days, the supernatants were pipetted, coded and stored at $-70^{\circ} \mathrm{C}$ until analysis. Cytokine dosage was determined in a blinded fashion by an independent lab using the "multibead array Milliplex Analyser v2.3 ${ }^{{ }^{\prime \prime}}$ system (Millipore Inc., MA, USA), which allows the simultaneous dosage of multiple cytokines. The ratio (SI) and the difference $(\varnothing)$ found between stimulated and unstimulated cultures were used to verify the result.

$$
\begin{aligned}
& \text { SI }=\text { value of stimulated cultures } \\
& \text { Value of unstimulated cultures } \\
& \varnothing=\text { value of stimulated cultures - value of } \\
& \text { unstimulated cultures }
\end{aligned}
$$

\section{Detection of $3 \mathrm{H}$ thymidine}

Detection of the response to pokeweed was made by comparing the incorporation of $3 \mathrm{H}$ thymidine in stimulated and unstimulated cultures in a manner similar to that performed with cytokines. The average of the stimulated cultures in triplicate was compared with that of unstimulated cultures in triplicate for each patient. The detection of $3 \mathrm{H}$ thymidine was made with the help of a scintillation counter (Betaplate, Wallac, Finland).

\section{Statistical Analysis}

The non-parametric Mann-Whitney U Test (GraphPad Prism ${ }^{\circledast}$ software, version 3.0) was used for statistical evaluation, and the results were considered statistically significant when $P<0.05$. For the statistical calculations, all values found below the capability of detection of the method were substituted for the minimum average value for the detection of each cytokine. The sensitivity, specificity and accuracy of the methods were calculated.

\section{RESULTS \\ Patch test}

All patients in the group of cases had PT positive for potassium dichromate. One of them was $(+)$, thirteen were $(++)$, and four were $(+++)$. In addition to chromium, eight patients were sensitized to carba mix, five were sensitized to thiuram mix and nickel sulfate, three were sensitized to cobalt and one to PPD mix, formaldehyde, nitrofurazone and quinoline.

All controls had PT negative for potassium dichromate. Five patients were sensitized to nickel sulfate, two were sensitized to cobalt, colophony, neomycin and fragrance mix, one was sensitized to benzocaine, chlorhexidine, ethylenediamine, nail enamel, formaldehyde, nitrofurazone, thimerosal, ammonium thioglycolate and thiuram mix.

\section{Detection of $3 \mathrm{H}$ thymidine}

Both groups showed a similar $(P=0.2185)$ and adequate proliferative response $(P<0.0001)$ when stimulated with pokeweed.

\section{Detection of cytokines}

The chromium stimulus induced the production of IFN-y in both cases $(P=0.0031)$ and controls $(P$ $=0.0102)$. The SI and $\varnothing$ were similar between the groups ( $P=0.3641$ and 0.8912 , respectively).

The addition of chromium led to increased production of IL-2 in the case group but not in the control group, $P=0.0012$ and 1 , respectively. The SI and $\varnothing$ found with IL-2 were different between cases and controls, $P=0.0007$ and 0.0010 , respectively. Using cut-off values above 1 for SI and values greater than zero for delta, the sensitivity, specificity and accuracy of IL-2 in the diagnosis of chromium-induced ACD were $72 \%, 95 \%$ and $84 \%$ for both the SI and delta.

There was no difference between the IL- 4 values detected in basal and stimulated cultures in the case group, $P=0.3113$, or in the control group, $P=$ 0.7703 . The values obtained through both the ratio 
and difference between chromium-stimulated and basal cultures in the case group were not different from those found in the control group, $P=0.5234$ and 0.3949 , respectively.

Chromium increased the expression of IL-5 in the case group $(P=0.0041)$, but not in the control group $(P=0.6491)$. The SI and $\varnothing$ values found with IL5 were similar for cases and controls, $P=0.0726$ and 0.1440 , respectively.

There was a reduction in the IL-10 values detected in chromium-stimulated cultures in both the case and control groups, $P<0.0001$ and 0.0007 , respectively. The SI and $\varnothing$ values were similar for cases and controls, $P=0.1966$ and 0.3087 , respectively.

The addition of chromium did not significantly alter the expression of IL-12 in stimulated cultures compared to unstimulated cultures in both the case and control groups, $P=0.3038$ and 0.5891 , respectively. The SI values found in the case group were higher than those found in the control group $(P=0.0298)$; however, the $\varnothing$ values were not $(P=0.05)$.

There was a greater production of IL-13 in the case cultures stimulated with chromium, but not in the cultures of the control group, $P<0.0001$ and 0.3885 , respectively (Graphs 1 and 2). Both the SI and $\varnothing$ values were higher in the case group compared to controls, $P=0.0018$ and 0.0014 , respectively (Graphs 3 and 4). Using values above 1.5 for SI and delta, we obtained a sensitivity, specificity and accuracy of $83 \%$, $68 \%$ and $76 \%$ for SI and $72 \%, 89 \%$ and $81 \%$ for delta, respectively.

The chromium stimulus given to the case and control cultures did not alter the expression of IL-17, $P$ $=0.7627$ and 0.9875 , respectively. We could not calculate whether there was a difference between the stimulated and unstimulated cultures in both groups regarding the values obtained through ratio and difference.

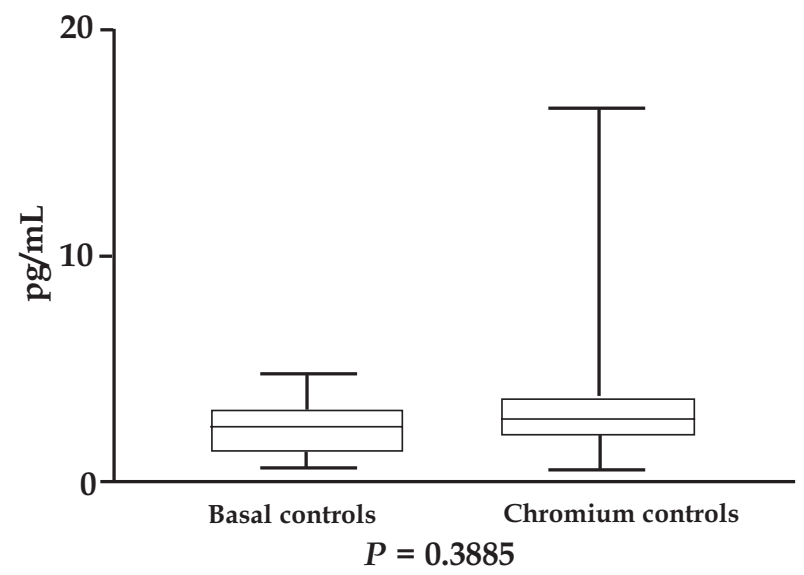

GrAPH 1: Detection of IL-13 in 2-day basal and chromium-stimulated cultures in the control group

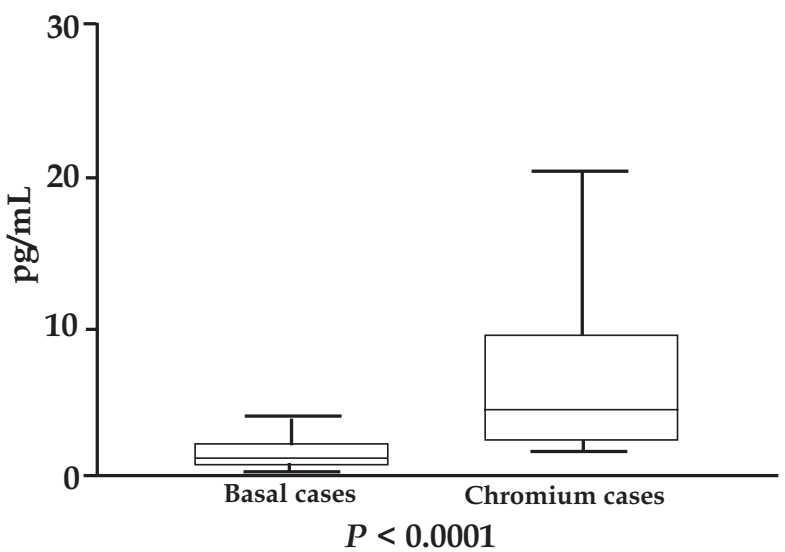

GRAPH 2: Detection of IL-13 in basal and chromium-stimulated cultures in the case group

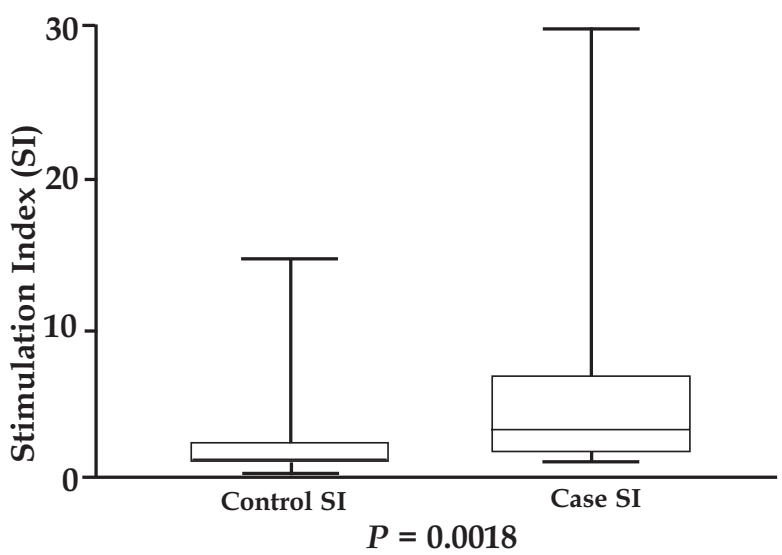

GRAPH 3: Stimulation indexes found with IL-13 in 2-day cultures from case and control groups

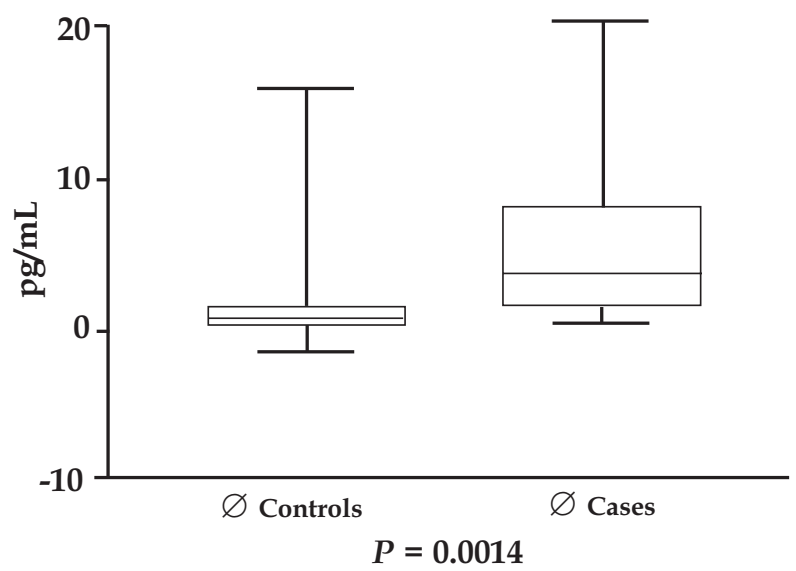

GrAPH 4: Difference in the detection of IL-13 between 2-day stimulated and basal cultures from case and control groups 
The addition of chromium to the case and control cultures did not alter the production of RANTES, $P=0.4108$ and 0.0541 , respectively. The SI and $\varnothing$ values found in the case group were similar to those found in the control group, $P=0.4942$ and 0.4752 , respectively.

\section{DISCUSSION}

About 6-7 days are necessary to analyze antigen-specific lymphocyte proliferation by marking the cells with thymidine, since the antigens initially affect only a few specific lymphocytes present in the culture and these lymphocytes activate the others, generating a response that is likely to be detected by this mehtod..$^{15}$ Detection of the results through cytokine happens earlier, since cell activation occurs before proliferation.

When choosing the cytokines that we used in this study, we kept in mind the fact that all of them may be involved in the immune response to a hapten stimulus, produced by effector memory lymphocytes in sensitized individuals whose regulatory cells do not work as those of individuals who do not develop ACD. ${ }^{2}$

The model we used presents conditions that are obviously different from those found "in vivo" in the epidermis, dermis, and lymphoid tissue of chromiumsensitized individuals. However, we are dealing with some cells of the peripheral blood of patients who are allergic or non-allergic to chromium and, thanks to the technique employed, the cells that predominate among these cells are lymphocytes, which may be hapten-specific and which should produce cytokines when in contact with chromium in order to produce an inflammatory response. We do not know if all the new concepts of the pathogenesis of ACD, resulting from studies in murine models, apply to the human species; however, the fact that NK, CD4 and CD8 T cells are effector cells in ACD led us to choose some cytokines. We chose IFN-y, IL-2, IL-4, IL-5, IL-10, IL12, IL-13, IL-17 and RANTES. Among the 9 cytokines that we studied, the chromium stimulus did not increase the production of IL-4, IL-10, IL-12, IL-17 or RANTES in allergic patients, but it stimulated the production of IFN-y, IL-5, IL-2 and IL-13.

In the case of IFN-y, there was increased production in the case group, but also in the control group. Therefore, since the ratio and difference between the values of basal and stimulated cultures of cases were statistically similar to those of controls, we discarded the possibility of using this cytokine in the diagnostic test of chromium-induced ACD in 2-day lymphocyte cultures. Minang et al. have also not found a difference in the production of IFN-y between 40-hour cultures from individuals allergic and nonallergic to chromium. ${ }^{16}$ However, Lindemann et al. reported higher production of IFN-y in 48-hour cul- tures from patients allergic to chromium compared to controls. ${ }^{17}$ Similarly to Lindemann et al., Trattner et al. found a difference in IFN-y production. These researchers performed 24-hour cultures stimulated with potassium dichromate and phytohemagglutinin, a non-specific activator of $T$ cells supposedly used to initiate and expand the reaction. ${ }^{17,18}$

The results of IFN-y production in short-term cultures are somewhat controversial, indicating that if IFN-y production is increased, this is not easily observed, which makes the method not so useful for the diagnosis of chromium-induced ACD. In a similar study, which used the same blood sample of the patients in this study and which followed the same method but with an incubation period of 6 days, we were able to demonstrate that IFN-y can help distinguish patients who are allergic to chromium from patients who are not. The sensitivity, specificity and accuracy found were $83 \%, 63 \%$ and $73 \%$ with the SI and $72 \%, 68 \%$ and $70 \%$ with the $\varnothing$, respectively. ${ }^{14}$

IL-5 production was increased in stimulated cultures from cases, but not from controls in our study. However, the SI and delta values found in cases and controls were similar, making this dosage useless to differentiate cases from controls. In the literature, nonetheless, Moed et al. and Martins et al. found an increase in IL-5 production in cultures stimulated with chromium. The first found a sensitivity of $100 \%$ and specificity of $86 \%$ in 6-day cultures, but in a few patients and in cultures costimulated with some cytokines. The latter found a sensitivity, specificity and accuracy of $72 \%, 79 \%$ and $76 \%$ with the SI and $56 \%, 89 \%$ and $73 \%$ with the delta. ${ }^{14,19}$

In the previous work by Martins et al. with 6day cultures, it was concluded that we could be able to use the measurement of IL-13 expression to replace PT in the diagnosis of chromium-induced ACD because of its high sensitivity, specificity and accuracy, which were $89 \%, 89 \%$ and $89 \%$ for the SI, respectively, and $94 \%, 89 \%$ and $92 \%$ for the delta. ${ }^{14}$ This study make us think that this alleged possibility does not apply to 2-day cultures, for the sensitivity, specificity and accuracy of $83 \%, 68 \%$ and $76 \%$ for the SI and $72 \%, 89 \%$ and $81 \%$ for the delta, respectively, were more modest. However, they are an alternative to 6day cultures or to the PT when this one cannot be performed.

IL-13 was the cytokine which generated better stimulation indexes. It is true, though, that we lowered sensitivity ( $83 \%$ for SI and $72 \%$ for delta), specificity (68\% for SI and $89 \%$ for delta) and accuracy $(76 \%$ for SI and $81 \%$ for delta) in relation to 6-day cultures. ${ }^{14}$ The results with 6-day cultures were amazing, and the confirmed increase of this cytokine in 2-day cultures leads us to think that this cytokine is very important 
in the emergence of ACD inflammation. Minang et al. also observed an increase in this cytokine in cultures of short-duration (40 hours) from patients allergic to chromium. ${ }^{16}$ IL-13 also showed to be promising as a diagnostic test with other antigens, in addition to helping distinguish allergic reactions from irritant reactions in patch tests. ${ }^{20,21}$

We can also add IL-2 as promising for the diagnosis of chromium-induced ACD, particularly in 2-day cultures. Although we have obtained sensitivity, specificity and accuracy results of $72 \%$ for both the SI and delta, there was a great improvement compared to what happened in 6-day cultures in the study by Martins et al. If these indexes do not allow us to think of replacing PT, they at least enable us to use IL-2 detection as a diagnostic aid in 2-day cultures, but not 6-day cultures, for patients with chromium-induced ACD. ${ }^{14}$

We know that IL-2 is very important to clonal expansion, which should occur more intensively in the early elicitation phase, which would possibly explain the increase in 2-day cultures in relation to 6day cultures under the same conditions. In the study involving 6-day cultures, there was no IL-2 increase in the stimulated cultures in cases or controls. There was only a difference in the SI but not in the delta. Minang et al., who used 20-hour cultures (ELISpot) and 40hour cultures (ELISA), also found an increase in the production of IL-2 in stimulated cultures; however, Lindemann et al. did not find this increase in 48-hour cultures. ${ }^{16,17}$

IL-4 was not stimulated by chromium in cultures from cases and controls in this study. The same occurred in short-term cultures by Minang et al. and Lindemann et al. and in 6-day cultures performed by our group, which makes it clear that this cytokine does not allow the diagnosis of ACD through the technique employed. ${ }^{16,17}$

With IL-10, the stimulus brought a decrease in production in both the case and control groups, with no difference in the diagnostic indexes. We repeated in the 2-day cultures what had occurred in 6-day cultures. Likewise, we conjecture that there has been some unknown, but non-specific factor that led chromium to lower the production of IL-10 by suppressor lymphocytes.

The production of IL-12 did not increase with the chromium stimulus in any of the groups, but there was a statistical difference for the SI. This phenomenon was also observed in the SI and $\varnothing$ values in 6-day cultures and resulted from the statistically non-significant increase in the means and medians of the SI of stimulated cultures combined with the also non-significant reduction of unstimulated cultures. Since it involves the ratio and difference between these values, the mathematical difference was observed. Lindemann $e t$ al. did not find an increased expression of IL-12 in 48-hour cultures from patients allergic to chromium. ${ }^{17}$ Therefore, if there is an increase, it is subtle, making this cytokine inadequate to differentiate patients with allergy to chromium using this method.

In the detection of IL-17, the results obtained with 2-day cultures corroborate the results found in 6day cultures by Martins et al., that is, there was no increase in IL-17 production in stimulated cultures in the case group or in the control group, and the diagnostic indexes were not statistically evaluated. ${ }^{14}$ Although IL-17 has a role in ACD, it was not useful in the diagnostic test.

In the case of RANTES, there was no increase with the stimulus in either group nor statistical differences in the diagnostic indexes. In the work by Martins et al., which involved 6-day cultures, chromium stimulated the production of this chemokine in both cases and controls in a similar manner; therefore, it also proved useless in the diagnostic detection. ${ }^{14}$

\section{CONCLUSIONS}

In this study involving two-day cultures, we concluded that the detection of IL-13 may be useful in the diagnosis of allergy to chromium in two-day cultures, as well as the detection of IL-2. None of the other cytokines studied showed results that could distinguish patients allergic to chromium from patients not allergic to chromium when using two-day cultures. Six-day cultures, in general, showed to be superior to 2-day cultures for this purpose. 


\section{REFERENCES}

1. Peiser M, Tralau T, Heidler J, Api AM, Arts JH, Basketter DA,et al. Allergic contact dermatitis: epidemiology, molecular mechanisms, in vitro methods and regulatory aspects. Current knowledge assembled at an international workshop at BfR, Germany. Cell Mol Life Sci.2012;69:763-81.

2. Martins LEAM, Reis VMS. Immunopathology of allergic contact dermatitis.An Bras Dermatol.2011;86:419-33.

3. Martins LE, Duarte AJ, Aoki V, Nunes RS, Ogusuku S, Reis VM. Lymphocyte proliferation testing in chromium allergic contact dermatitis.ClinExpDermatol.2008;33:472-7.

4. Uter W, Aberer W, Armario-Hita JC, Fernandez-Vozmediano JM, Ayala F, Balato A,et al. Current patch test results with the European baseline series and extensions to it from the 'European Surveillance System on Contact Allergy' network, 2007-2008. Contact Dermatitis.2012;67:9-19.

5. Lindberg M, Matura M. Patch testing. In: Johansen JD, Frosch P, Lepoittevin JP, editors.Contact dermatitis. 5th ed. Heidelberg: Springer; 2011. p. 439-64.

6. Wolf R, Davidovici B, Marcos B, Orion E. Lymphocyte transformation test in patients with allergic contact dermatitis. Contact Dermatitis. 2005;53:245

7. Kligman AM. A personal critique of diagnostic patch testing.Dermatology.1996; 14:35-40.

8. Duarte I, Lazzarini R, Buense R. Interference of the position of substances in an epicutaneous patch test battery with the occurrence of false-positive results. Am J Contact Dermat. 2002;13:125-32.

9. Brasch J, Henseler T, Aberer W, Bäuerle G, Frosch PJ, Fuchs T,et al. Reproducibility of patch tests. A multicenter study of synchronous left-versus right-sided patch tests by the German Contact Dermatitis Research Group. J Am Acad Dermatol. 1994;31:584-91.

10. Fisher T, Rystedt I. False-positive, follicular and irritant patch test reactions to metal salts. Contact Dermatitis.1985;12:93-8.

11. Beck MH, Wilkinson SM. Contact dermatitis: allergic. In:Burns T, Breathnach S, Cox N, Griffiths C. Rook's Textbook of Dermatology. 7th ed. Oxford: Blackwell Science Publishers; 2004. p.20.1-124.

12. Andersen KE, White IR, Goossens A. Allergens from the European Baseline Series. In: Johansen JD, Frosch PJ, Lepoittevin JR, editors. Contact Dermatitis. Heidelberg: Springer; 2011. p. 545-90.

13. Sanchez APG, Maruta CW, Sato MN, Ribeiro RL, Zomignan CA, Nunes RS, et al. Study on lymphocyte proliferation in nickel-sensitive patients. An Bras Dermatol. 2005;80:149-58.

14. Martins LEAMM, Reis VMS. IL-13: a marker of chromium contact allergy. J EurAcadDermatolVenereol.2012.doi: 10.1111/jdv.12005. [Epub ahead of print]

15. Frank MM, Austen KF, Claman HN, Unanue ER. Samter's immunologic diseases. 5th ed. New York: Little, Brown; 1995.

16. Minang JT, Areström I, Troye-Blomberg M, Lundeberg L, Ahlborg N. Nickel, cobalt, chromium, palladium and gold induced a mixed Th1and Th2 type cytokine response in vitro in subjects with contact allergy to the respective metals. Clin Exp Immunol. 2006;146:417-26.

17. Lindemann M, Rietschel F, Zabel M, Grosse-Wilde $H$. Detection of chromium allergy by cellular in vitro methods. Clin Exp Allergy. 2008;38:1468-75.

18. Trattner A, Akerman L, Lapidoth M, Klein T, Weiss H, Ben Chaim B, et al. Use of in vitro release of interferon gama in the diagnosis of contact allergy to potassium dichromate a controlled study. Contact Dermatitis. 2003;48:191-3.

19. Moed H, Von Blomberg M, Bruynzeel DP, Scheper R, Gibbs S, Rustemeyer T. Improved detection of allergen- specific T-cell responses in allergic contact dermatitis through the addition of "cytokine cocktails". Exp Dermatol. 2005;14:634-40.

20. Wahlkvist H, Masjedi K, Gruvberger B, Zuber B, Karlberg AT, Bruze M,et al. The lipophilic haptenparthenolide induces interferon- $\gamma$ and interleukin-13 production by peripheral blood-derived $\mathrm{CD} 8+\mathrm{T}$ cells from contact allergic subjects in vitro. $\mathrm{Br} \mathrm{J}$ Dermatol. 2008;158:70-7.

21. Morhenn VB, Chang E-Y, Rheins LA.A noninvasive method for quantifying and distinguishing inflammatory skin reactions. J Am Acad Dermatol. 1999;41:687-92.

\author{
MAILING ADDRESS: \\ Vitor Manoel Silva dos Reis \\ Rua Teodoro Sampaio, 352 - cj36 \\ 05406-000 - São Paulo - SP \\ Brazil \\ E-mail: vitoreis76@hotmail.com
}

How to cite this article: Martins LEAM, Reis VMS. Cytokine detection for the diagnosis of chromium allergy. An Bras Dermatol. 2013;88(5):719-25. 